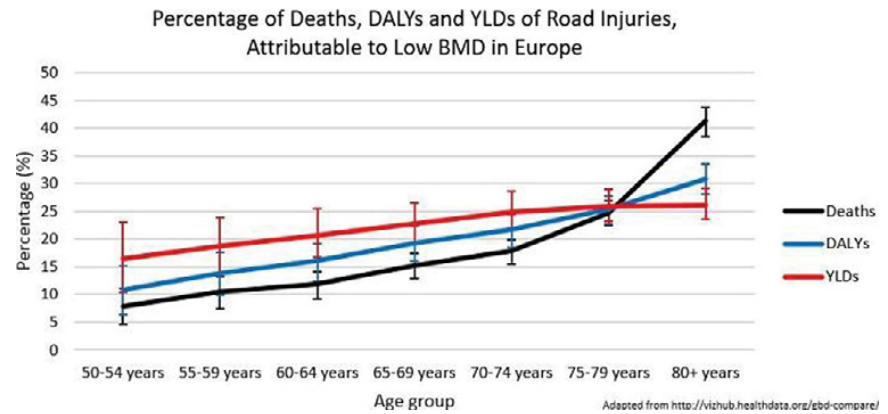

References:

[1] Forouzanfar et al. Lancet 2016.

[2] Johnell et al. JBMR 2005.

Disclosure of Interest: None declared

DOI: 10.1136/annrheumdis-2017-eular.3278

\section{AB1103 THE IMPORTANCE OF ADEQUATE SCREENING TO AVOID FALSE POSITIVES IN THE DIAGNOSIS OF RHEUMATOID ARTHRITIS}

P. Santos-Moreno ${ }^{1}$, D. Gomez ${ }^{1}$, E. Castillo ${ }^{1}$, R. Giraldo ${ }^{1}$, G. Ballesteros ${ }^{1}$, L. Villarreal ${ }^{2}$, D. Buitrago-Garcia ${ }^{3}$, J. Bello ${ }^{3} \cdot{ }^{1}$ Rheumatology; ${ }^{2}$ Psychology and processes; ${ }^{3}$ Epidemiology, Biomab, Center for Rheumatoid Arthritis, Bogota, Bogota, Colombia

Background: The lack of expertise and skills in the diagnosis of in rheumatoid arthritis (RA) in primary level of Colombian medical centers can cause misdiagnosis of rheumatic diseases. Due to this issue in a specialized center in RA we stablished a multidisciplinary model and a strict disease management algorithm to diagnose properly our patients; as a consequence we have achieved the accurate diagnosis of great proportion of patients that were false positives diagnosed initially as RA.

Objectives: The aim of this study was to show effectiveness and accuracy of a screening method to avoid false-positive diagnosis of RA in a cohort of patients with supposed diagnosis of RA.

Methods: During two years we evaluated patients with presumptive diagnosis of RA. We conducted a cross-sectional study; we included patients who were referred from primary care centers to a RA specialized center in a 24 month period with presumptive diagnosis of (RA). Each patient was evaluated to confirm or rule-out diagnosis of RA as follows: a rheumatologist fulfilled a complete medical record, including joint counts; it was assessed rheumatoid factor and anti-citrullinated antibodies, and other laboratories depending on each case. Also were made x-rays of hands and feet, and in some cases of persistent doubt about the diagnosis was requested comparative MRI of hands or/and feet. Descriptive epidemiology was perfomed.

Results: Between 2015 and 20166813 patients were evaluated in our specialized center, in $76 \%$ of cases RA was confirmed, the remaining 1593 patients (24\%) had a wrong diagnosis of RA; of these misdiagnosed patients, $(87 \%)$ were female, and $205(13 \%)$ male, with an average age of $62 \pm 12$ years. Between differential diagnosis which were found in this cohort of misdiagnosed patients: osteoarthritis in 849 patients (63.3\%), Sjögren syndrome (7\%) Systemic lupus erythematosus (6\%) the remaining $30 \%$ of patients had conditions such as gout, psoriasis, osteoporosis, myalgia, soft tissue diseases among others. The majority of patients with wrong diagnosis took DMARDs $(23 \%)$, calcium (11\%), biologics $(10 \%)$ acetaminophen $(9 \%)$, neuropathy medications $(7 \%)$, acetaminophen plus opioids $(5 \%)$, osteoporosis medications $(5 \%)$, opioids $(4 \%)$, glucosamine $(4 \%)$, diacerein $(3 \%)$, the remaining patients took medications such as NSAID, glucosamine, antigout agents, gastritis drugs, among others.

Conclusions: The results of this program show that almost $25 \%$ patients with presumptive RA diagnosis are misdiagnosed; this is evidence that can be extrapolated to primary care centers in Colombia. The most important cofounding diagnosis was osteoarthritis and many patients were receiving DMARDs for treatment. For this reason there is an urgent need of education strategies for primary care physicians and the implementation of centers of excellence in RA, in order to conduct a proper diagnose and avoid clinical and health economics consequences of misdiagnosis.

Disclosure of Interest: None declared

DOI: 10.1136/annrheumdis-2017-eular.5249

\section{AB1104 COST-EFFECTIVENES ANALYSIS OF TNF INHIBITORS USE COMPARED TO DMARDS IN THE FATAL AND NONFATAL ACUTE CORONARY ISCHEMIC EVENT}

R.K.S. Gomes ${ }^{1}$, J.Y.K. Viscondi ${ }^{2}$, M.R.C. Nobre ${ }^{2}{ }^{1}{ }^{1}$ Center of specialty of the municipality of Blumenau and Brusque, Blumenau; ${ }^{2}$ São Paulo University, São Paulo, Brazil

Background: Epidemiological studies have established that rheumatoid arthritis is associated with an increase in cardiovascular disease ${ }^{1,2}$. The evaluation of tumour necrosis factor inhibitors (TNFi) on the reduction of the risk of acute myocardial infarction and death due to cardiovascular causes has shown promising results ${ }^{3}$. The economic evaluation for these outcomes are not established yet.

Objectives: To evaluate the cost-effectiveness of TNFi versus disease-modifying antirheumatic drugs (Dmards) to avoid a new case of acute ischemic heart disease and death in rheumatoid arthritis patients.

Methods: A cost-effectivenes analysis (CEA) was performed using a Markov model 6-month transition cycle, with time horizon of 30 years, under the Brazilian public healthcare system perspective. Costs are expressed in 2015 Reais and effectiveness measures are new cases of acute ischemic coronary disease and cardiovascular death.

Results: The average cost in 30 years of Dmards and TNFi was 14,291,105.28 and $96,151,873.86$ Reais, respectively. The incremental effectiveness was 2.69 cases of coronary artery disease and consequent incremental cost-effectiveness ratio (ICER) of 30,527,502.27 Reais per new cases avoided, while for cardiovascular death, incremental effectiveness was 1.33 and an ICER of 61,634,231,69 Reais per new cases avoided. The univariate analysis identified that the most relevant parameter in the ICER on both outcomes was the TNFi drug. The sensitivity analysis established that, in order to, reach the amount of willingness to pay (WTP) per semester to avoid an acute myocardial infarction, the average cost of TNFi should be 1,337.47 Reais per case avoided and the average cost for the cardiovascular death avoidence sould be 954.22 Reais. All the analyzes performed establish an unfavorable relationship of the drug treatment strategy with TNFi.

Conclusions: The findings of the CEA among patients with rheumatoid arthritis for cardiovascular outcomes when compared to the strategy of TNFi drug treatment with the dominant strategy Dmards after the first 6 months of exposure point out an unfavorable relationship, surpassing the amount of expenses recommended by the Ministry of Health of Brazil in the year 2015.

\section{References:}

[1] Symmons DP, Gabriel SE. Epidemiology of CVD in rheumatic disease, with a focus on RA and SLE. Nat Rev Rheumatol. 2011;7(7):399-408.

[2] Bergström U, Jacobsson LT, Turesson C. Cardiovascular morbidity and mortality remain similar in two cohorts of patients with long-standing rheumatoid arthritis seen in 1978 and 1995 in Malmö, Sweden. Rheumatology (Oxford). 2009;48(12):1600-5.

[3] Dixon WG, Watson KD, Lunt M, Hyrich KL; British Society for Rheumatology Biologics Register Control Centre Consortium. Reduction in the incidence of myocardial infarction in patients with rheumatoid arthritis who respond to anti-tumor necrosis factor alpha therapy: results from the British Society for Rheumatology Biologics Register. Arthritis Rheum. 2007;56(9):2905-12.

Acknowledgements: Foundation for Research Support of the State of São Paulo, FAPESP 2013/12979-1.

Disclosure of Interest: None declared

DOI: 10.1136/annrheumdis-2017-eular.2124

\section{AB1105 FACTORS IMPACTING PATIENTS' DECISION TO START TREATMENT VARY BY RACE}

R. Cozmuta ${ }^{1}$, L. Fraenkel ${ }^{2} .{ }^{1}$ Rheumatology, Emory University, Atlanta; ${ }^{2}$ Yale University, New Haven, United States

Background: Previous research has found that young minority women tend to be more risk averse compared to their Caucasian counterparts. The reasons underlying these differences, however, are not understood.

Objectives: The objective of this study was to examine whether factors influencing perceived treatment importance vary by race.

Methods: Women between the ages of 20 and 45 completed a survey. The survey recorded sociodemographic data, trust in healthcare systems and beliefs in medications. It also included a hypothetical scenario in which subjects were asked to rate the importance of taking a medication for a patient with joint pain, migraines and fatigue that benefits $70 \%$ of people and is well tolerated except for the rare risk (1 per 100,000) of a neurologic disease that may cause weakness, trouble with vision and numbness. Associations between patient characteristics, medication beliefs, and trust with perceived importance of taking the medication were evaluated for each race. Variables found to be statistically significant were subsequently evaluated using multiple linear regression.

Results: 299 women completed the survey. Baseline characteristics by ethnicity are described in Table 1. Hispanic women had more negative medication beliefs than did Non-Hispanic Whites, and both Non-Hispanic Black and Hispanic

Table 1. Patient characteristics by race

\begin{tabular}{lcccc}
\hline Variable & Non-Hispanic White & Non-Hispanic Black & Hispanic & P value \\
\hline Age (mean, SD) & $34.1(7.2)$ & $35.2(8.4)$ & $32.8(8.6)$ & 0.1 \\
Poor self-reported health (\%) & 12 & 14 & 12 & 0.9 \\
Some college education (\%) & 75 & 61 & 43 & $<0.01$ \\
Income <12,000/year (\%) & 23 & 33 & 34 & 0.2 \\
Difficulty paying for meds (\%) & 68 & 58 & 60 & 0.3 \\
Medication beliefs (mean, SD) & $20.6(4.6)$ & $21.6(4.4)$ & $22.3(4.7)$ & 0.04 \\
Trust (mean, SD) & $27.9(5.5)$ & $28.6(5.7)$ & $29.1(5.5)$ & 0.3 \\
Hopeful (mean, SD) & $4.2(1.6)$ & $3.5(1.7)$ & $3.6(1.7)$ & $<0.01$ \\
Worried (mean, SD) & $4.6(1.6)$ & $4.8(1.8)$ & $5.1(1.6)$ & 0.2 \\
Important (mean, SD) & 5.3 & 5.6 & 5.5 & 0.3 \\
\hline
\end{tabular}


Table 2. Association of subject characteristics with perceived importance by race

\begin{tabular}{|c|c|c|c|}
\hline Variable & Non-Hispanic Whites & Non-Hispanic Blacks & Hispanics \\
\hline Medication beliefs ${ }^{1}$ & $-0.55(<0.01)$ & $-0.24(\mathbf{0 . 0 1})$ & $-0.08(0.46)$ \\
\hline Hopeful $^{1}$ & $0.54(<0.01)$ & $0.16(0.09)$ & $0.37(<0.01)$ \\
\hline Worried $^{1}$ & $-0.27(\mathbf{0 . 0 1 )}$ & $-0.02(0.87)$ & $0.05(0.66)$ \\
\hline Trust $^{1}$ & $0.22(0.02)$ & $0.33(\mathbf{0 . 0 1})$ & $0.17(0.11)$ \\
\hline Poor self-reported health ${ }^{2}$ & $1.66(0.10)$ & $1.19(0.25)$ & $-1.4(0.16)$ \\
\hline Some college education ${ }^{2}$ & $-1.16(0.25)$ & $1.9(0.06)$ & $0.65(0.52)$ \\
\hline Income $<12,000 /$ year $^{2}$ & $0.38(0.71)$ & $1.65(0.11)$ & $0.06(0.96)$ \\
\hline Difficulty paying for meds ${ }^{2}$ & $0.00(0.99)$ & $-2.05(0.04)$ & $-0.42(0.68)$ \\
\hline
\end{tabular}

${ }^{1}$ Correlation coefficient ( $p$ value); ${ }^{2}$ T-test ( $p$ value).

subjects had lower levels of hope compared to Non-Hispanic White subjects (difference between means $<0.05$ ). Associations between subject characteristics and perceived importance of taking the medication are presented in Table 2. In a multilinear regression model (including education, difficulty paying for medications, medication beliefs, trust, hope and worry), hope was associated with perceived importance of taking the medication in all three ethnic groups. Additional findings differed by race, with medication beliefs in Non-Hispanic White subjects; difficulty paying for medications in Non-Hispanic Black subjects, and worry in Hispanic subjects being associated with perceived importance of taking the medication.

Conclusions: Our findings confirm the important influence of emotion on decision making, and suggest that while hope is universally associated with perceived importance of taking a medication, other factors differed, highlighting differences in the decision making process across ethnic groups.

Disclosure of Interest: None declared

DOI: 10.1136/annrheumdis-2017-eular.2369

\section{AB1106 VERBAL PATIENT EDUCATION ON VACCINATION IN ADULTS WITH AUTOIMMUNE INFLAMMATORY RHEUMATIC DISEASES: IS IT ENOUGH TO IMPROVE VACCINATION RATES?}

R. Magro, M. Rogers, F. Camilleri. Rheumatology, Mater Dei Hospital, Msida, Malta

Background: Patients suffering from autoimmune inflammatory rheumatic diseases (AIIRD) are at increased risk of infections, due to the underlying condition and its treatment. A study on vaccination in AIIRD carried out at Mater Dei Hospital, Malta in 2015, showed that $37.9 \%$ of the patients received the influenza vaccine in the previous year and $8.3 \%$ had received the pneumococcal vaccine ever. Only $38.3 \%$ knew that they had an increased risk of infection because of their condition.

Objectives: The aim of the current study was to establish whether verbal education on vaccination, influenced patients' vaccination rates in the following year. A further aim was to determine whether the patients had a better understanding of their increased infection risk following verbal education.

Methods: The initial study carried out in 2015 consisted of a short face-to-face interview with 60 patients who suffered from autoimmune inflammatory rheumatic diseases. Following the interview, verbal information was given to the patients on their increased risk of infection, and the importance of vaccination, in particular the influenza and pneumococcal vaccine. After 1 year, the patients were contacted by means of a telephone call. They were interviewed with regards to their vaccination history and knowledge of their infection risk. Of the 60 patients, 2 had passed away. Therefore 58 patients were included in the study.

Results: The study included patients with a variety of AIIRD including rheumatoid arthrtitis, ankylosing spondylitis and psoriatic arthritis. $60.3 \%$ were females and the mean age was 63.1 years (range 25 to 82 years). Influenza vaccination rates in the previous year improved from $37.9 \%$ in 2015 to $41.4 \%$ in 2016 ( $p=0.704)$. Pneumococcal vaccination rates improved from $6.9 \%$ to $17.2 \%(p=0.086)$. On questioning the patients regarding their knowledge of increased infection risk, there was no improvement following the verbal education. In fact $37.9 \%$ of patients questioned in 2015 , and $34.5 \%$ in 2016 knew that they were at increased risk of infection because of their condition. 33\% of patients studied in 2016 could recall that they had been advised to take vaccination because of their underlying condition or treatment. This improved from $7 \%$ in $2015(p<0.005)$. The proportion of patients who could recall the vaccination advice given was significantly higher in those below 61 years $(p<0.005)$. However, there were no significant gender difference $(p=0.147)$.

Conclusions: Verbal education resulted in more patients receiving the pneumococcal vaccination (although not statistically significant because of the small number involved). It also resulted in a significant increase in the number of patients who were able to recall that they were adviced to take vaccination because of their condition. However, only $33 \%$ could recall this after a year. Verbal education did not effect the number of patients who took the influenza vaccine and the patients' knowledge of their increased infection risk.

This study highlights that verbal education helps to improve vaccination rates in autoimmune inflammatory rheumatic diseases. However, the advice given tends to be forgotten by the majority of patients after one year. Thus repeated verbal education is necessary, particularly in elderly patients. Moreover other methods of education, such as written information in the form of leaflets, may be useful.

Disclosure of Interest: None declared

DOI: 10.1136/annrheumdis-2017-eular.1962

\section{AB1107 ASSESSMENT OF AGREEMENT BETWEEN SELF-REPORT INFLAMMATORY ARTHRITIS SYMPTOMS AND CORRESPONDING GP DIAGNOSIS IN PATIENTS WITH PMR}

S.L. Hider ${ }^{1,2}$, M. Blagojevic-Bucknall ${ }^{2}$, S. Muller ${ }^{2}$, K. Clarkson ${ }^{2}$, C. Mallen ${ }^{2}$. ${ }^{1}$ Haywood Academic Rheumatology Centre, Haywood Hospital, Staffordshire; ${ }^{2}$ Arthritis Research UK Primary Care Centre, Keele University, Keele, United Kingdom

Background: Polymyalgia rheumatica (PMR) is the commonest inflammatory condition among those aged 50 years and over, with a lifetime risk of $2.4 \%$ in females and $1.7 \%$ in males. Seronegative rheumatoid arthritis is an important differential diagnosis. It is thought a proportion of PMR patients develop an inflammatory arthritis, although prevalence estimates vary.

Objectives: To determine the proportion of PMR patients (1) reporting symptoms of possible inflammatory arthritis using a validated self-report inflammatory arthritis algorithm; and (2) who subsequently receive a diagnosis of inflammatory arthritis in their primary care medical records.

Methods: A cross-sectional questionnaire study was developed to investigate impact of PMR in primary care patients. All eligible adults aged $\geq 50$ years, registered with one of 150 participating practices with a first ever Read coded diagnosis of PMR in the last 3 years were mailed a questionnaire including items about socio-demographics, PMR characteristics, joint problems and comorbid conditions. Self-reported inflammatory arthritis (IA) was defined using a validated scoring algorithm (1) with positive responses coded as 1 and negative responses as -1 ; a score $>0$ is regarded as predictive of IA. IA score was calculated for those patients that had complete data on all required variables. A list of Read Codes relating to inflammatory arthritis, compiled by a rheumatologist and a GP, were searched for in primary care medical records of consenting respondents. Period of interest was from date of first ever PMR diagnosis in the medical records until survey mail-out.

Results: 704 eligible patients were identified and sent a questionnaire, with 550 $(78 \%)$ responding. Responders and non-responders did not differ significantly by age or gender. Medical records could be obtained for 385 patients, of whom 310 completed the questionnaire fully and were included in the analysis. IA score ranged from -8.4 to 2.5 , with $21(7 \%)$ patients being classed as having a positive IA score. 8 out of 310 patients had at least one inflammatory arthritis Read code recorded from date of first diagnosis of PMR in their medical record, although only 2 of these also fulfilled the self-report IA criteria.

Conclusions: In this PMR cohort the proportion subsequently diagnosed with an inflammatory arthritis was low $(2.5 \%)$ although more patients reported inflammatory symptoms. The agreement between self-reported inflammatory symptoms and Read Code diagnosed inflammatory arthritis was low.

References:

[1] Tavares R, Wells GA, Bykerk VP et al. A a parallel group cohort to determine the measurement properties of an early inflammatory arthritis detection tool. Rheumatology 2013;52:2077-2085.

Acknowledgements: This study represents independent research funded by the National Institute for Health Research (NIHR). CDM is funded by the National Institute for Health Research (NIHR) Collaborations for Leadership in Applied Health Research and Care West Midlands, the NIHR School for Primary Care Research and a NIHR Research Professorship in General Practice (NIHR-RP2014-04-026). The views expressed are those of the authors and not necessarily those of the NHS, NIHR or the Department of Health.

Disclosure of Interest: None declared

DOI: 10.1136/annrheumdis-2017-eular.5271

\section{AB1108 HANDLING OF DE NOVO ARTHRITIS IN ADULTS BY GENERAL PRACTITIONERS: A SURVEY IN A FRENCH COHORT}

S. Lecauchois ${ }^{1}$, S. Gentile ${ }^{2}$, P. Champsaur ${ }^{3}$, J.-P. Mattei ${ }^{4}$, L. Dillinger ${ }^{5}$, V. Goeb ${ }^{6}$ S. Guis ${ }^{4,7}$. ${ }^{1}$ Aix Marseille Université, AP-HM, Marseille Cedex 9, France; ${ }^{2}$ Public Health; ${ }^{3}$ Radiology; ${ }^{4}$ Rheumatology, Aix Marseille Université, AP-HM, Marseille Cedex 9; 5 General Practitioner, Aix-en-Provence;

${ }^{6}$ Rheumatology, CHU Amiens, Université de Picardie Jules Verne, Amiens; ${ }^{7} \mathrm{Crmbm}$-Cemerem UMR 7339, CNRS, Aix Marseille Université, Marseille, France

Background: A guide of good clinical practice regarding de novo arthritis in adults has been edited.

Objectives: The aim of this work is to identify whether the diagnostic and therapeutic tools proposed by general practitioners (GPs) to adults with a de novo arthritis is in line with the corresponding recommendations.

Methods: A questionnaire regarding the characteristics and the handling of de novo arthritis in adults has been submitted to 300 GPs chosen randomly from a French cohort database. The study period was from February 2015 to February 2016. Fifty seven GPs participated in the study (19\%).

Results: No particular specificity was identified in the GP group. The age of patients ranged between 40 and 75 yo. The prevalence of arthritis was less than $5 \%$ among the GPs patients. The relative number of each category of arthritis was rheumatoid arthritis (29\%), spondyloarthritis (14\%), PPR $(8 \%)$, other kinds of microcrystalline arthritis: chondrocalcinosis, (38.4\%), gout (11,6\%).

Joints with mechanic degenerative effusion related to osteo-arthritis might be misdiagnosed as an inflammatory arthritis.

The knee was the most frequently involved joint in arthritis. The initial paraclinical 